\title{
AN UNCOUPLED OSCILLATOR MODEL OF THE HOFFMANN REFLEX
}

\author{
Stephen J. Dorgan ${ }^{\dagger \ddagger}$, Member IEEE and Richard B. Reilly ${ }^{\dagger}$, Member IEEE \\ ${ }^{\dagger}$ Department of Electronic and Electrical Engineering, University College Dublin, \\ National University of Ireland, Dublin 4, Ireland. \\ ${ }^{\ddagger}$ Institute of Automatic Control Engineering, Technical University of Munich, 80333 Munich, Germany. \\ E-mail : s.j.dorgan@ieee.org
}

\begin{abstract}
A simple, new, mathematical model for the Hoffmann reflex is outlined. This model has been developed by considering phase synchronisation of the underlying neuromuscular processes by the electrical stimulation pulse. The novel approach used results in an analytical representation of the muscle's EMG response to a stimulation pulse. With this model, studies to provide insight into, and assessment of, the Hoffmann Reflex in response to electrical stimuli delivered to the popliteal fossa at various intensity levels may be carried out. Indices based on the parameters of this model may be developed to provide objective Hoffmann Reflex comparisons for the clinical assessment of reflexes in the diagnosis of nerve damage.
\end{abstract}

\section{INTRODUCTION}

Voluntary movement is brought about by the contraction of skeletal muscle fibre. Within the human nervous system many feedback and feed forward mechanisms exist to control skeletal muscle tension. Feed-forward mechanisms attempt to provide the appropriate level of neural activation to the muscle while feedback loops, via the reflex pathways, provide information regarding the real state of the muscular length and tension to the spinal motor neurone pools.

Electrical stimulation of the posterior tibial nerve, at the popliteal fossa, results in contraction of the soleus muscle. Response to this stimulus may be monitored using electromyography (EMG) which measures the summation of individual muscle fibre action potentials. The response elicited consists of a component of short latency and/or a second component of longer latency, as in Fig. 1. The early response is due to direct activation of the motor neurones and has a latency of about $5 \mathrm{~ms}$ and is known as the $M$-wave. The later response, occurring about $35 \mathrm{~ms}$ post-stimulus, is evoked at lower stimulation levels and is the reflex action known as the Hoffmann Reflex (or H-reflex) [1] [2] [3]. The H-reflex occurs as the posterior tibial nerve, is a common pathway for the motor and sensory nerves, both of which are being stimulated. While equivalent in many respects to the monosynaptic stretch reflex, the H-reflex bypasses the muscle spindle's elicitation of sensory neural information by direct electrical stimulation of the sensory nerves.

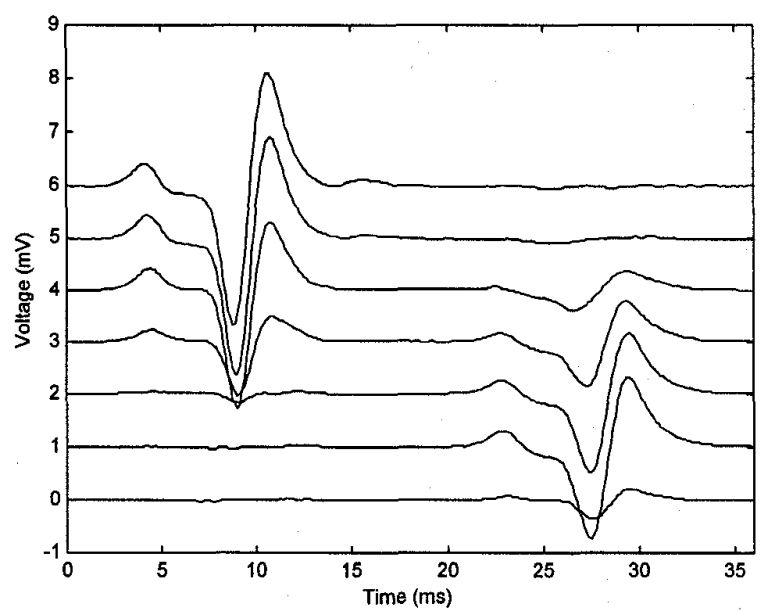

Fig. 1 Hoffmann responses for a standing upright subject. For clarity each test is shifted $1 \mathrm{mV}$ more along the Voltage axis.

The Hoffmann reflex was first observed early this century and its clinical importance is now well established [1]-[5]. It is an adequate method for studying the strength of the short latency loop in man. The clinical importance of reflexes is in the diagnosis of nerve damage. If the reflex response is exaggerated, it may be as a result of damage to the spinal cord. If the reflex response is unobservable it may indicate damage to the reflex centres in the spinal cord or damage to the peripheral nerves.

Fig. 1 shows the modulation of the H-reflex, observed in experiment, as the stimulus intensity is increased. The Hreflex initially increases with increasing stimulus intensity. Once the $\mathrm{M}$-wave is elicited, a further increase in stimulus intensity causes the H-reflex to decrease. Amongst possible causes for the decrease of the H-reflex with increasing stimulus, are [1]:

- Collision of the reflex impulse with the motor nerve activity in the opposite direction, antidromic activity.

- Refractioness of the axon hillock, the region of the neurone which becomes the axon, although it is argued that this is a secondary activity to the antidromic process [1]. 


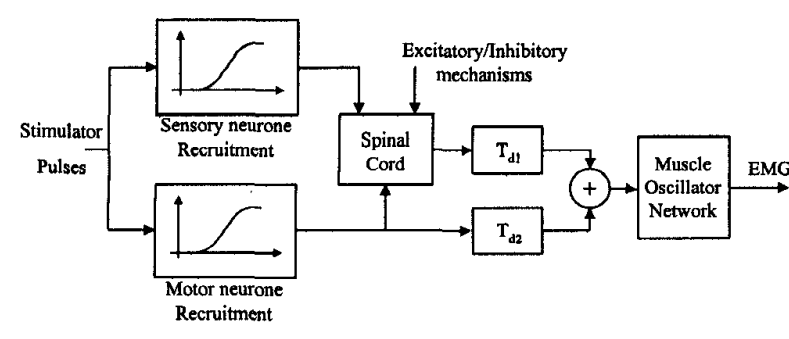

Fig. 2 Macroscopic structure of the model used here.

\section{DATA ACQUISITION HARDWARE AND SOFTWARE}

Custom hardware and software was developed for the acquisition of EMG activity following the delivery of a stimulation pulse at the popliteal fossa [6]. The test was initiated by a hand-held switch, causing a stimulation pulse to be generated by an electrical stimulator and simultaneously triggering the EMG recording procedure, as described in [6].

The software controlled EMG data acquisition at a sampling frequency of $10 \mathrm{kHz}$. The software is also capable of repetitive acquisition at given points in the gait cycle to investigate the modulation of the H-reflex during gait in subjects [7]. Software has also been developed to automate the generation of graphics and the analysis of results.

\section{MACROSCOPIC MODEL STRUCTURE}

The macroscopic structure of the model developed here is shown in Fig. 2. While based on the underlying physiological structure and its properties, the model is simple and therefore capable of providing easily accessible insight into the properties of the underlying system. The stimulation pulses recruit increasing numbers of sensory or motor neurones as the stimulation intensity (pulse width or pulse amplitude) is increased according to:

$$
r(t)=c_{1}+c_{2} \tanh \left(c_{3} \cdot a(t)-c_{4}\right)
$$

where $r(t)$ is the normalised number of neurones recruited, $a(t)$ is the simulation intensity and $c_{i} \in \mathfrak{R} \forall i$. Different parameter values are used in eqn. (1) to describe the sensory and motor neurone pools, in order to reflect their different recruitment profiles.

The interaction at the spinal cord is simply modelled by the equation:

$$
r_{r}(t)=c_{5} \cdot r_{s}(t) \cdot\left[1-r_{m}(t)\right]
$$

where $r_{s}(t), r_{r}(t)$ and $r_{m}(t)$ are the normalised number of motor units recruited in the sensory, motor, and H-reflex motor neurone pools, respectively. Eqn. (2) captures in a simple way the number of motor neurones the H-reflex loop may recruit at a spinal level as a function of both sensory and motor neurone recruitment levels and antidromic activity.

\section{OSCILlator MODEL OF EMG}

One of the features of muscle contraction elicited by surface electrical stimulation is that the activation of motor units is synchronous. Individual muscle fibre action potentials combine to form a motor unit action potential (MUAP), and it is the summation of MUAPs that produces the EMG signal measured at the electrodes. However, each MUAP may be considered as an oscillator switched on for one cycle in response to the neural action potential arriving at the motor end plate. Therefore, a simple EMG model composed of linear oscillators is presented here to simulate the synchronisation of phases, in the muscle's motor unit population in response to the synchronous activation by the $\mathrm{M}$-wave and $\mathrm{H}$-wave motor stimuli. There are differences in the travelling times of neural action potentials, motor endplate locations, neuromuscular junction delays and fibre conduction velocities, all of which contribute to a time dispersion effect in the MUAPs within the EMG signal. Therefore a probabilistic approach is taken to modelling this oscillator pool. Consider $-\sin (\omega t)$, where $\omega$ is a random variable with probability density function [8]

$$
p(\omega)=\frac{1}{\sigma \sqrt{2 \pi}} e^{-\frac{(\omega-\mu)^{2}}{2 \sigma^{2}}}
$$

The expected value of $\sin (\omega t)$ is

$$
\begin{aligned}
& y(t)=\int_{\omega=-\infty}^{\infty} \sin (\omega t) \cdot \frac{-1}{\sigma \sqrt{2 \pi}} e^{-\frac{(\omega-\mu)^{2}}{2 \sigma^{2}}} \cdot d \omega \\
& y(t)=\frac{-1}{2 j \sigma \sqrt{2 \pi}} \int_{\omega=-\infty}^{\infty}\left\{e^{j \omega t}-e^{-j \omega t}\right\} e^{-\frac{(\omega-\mu)^{2}}{2 \sigma^{2}}} \cdot d \omega
\end{aligned}
$$

Since $p(\omega)$ is a probability density function,

$$
\int_{-\infty}^{\infty} p(\omega) d \omega=1
$$

independent of $\mu$ and $\sigma$. Labelling part of RHS of eqn. (4) $I_{1}$

$$
I_{1}=\frac{1}{\sigma \sqrt{2 \pi}} \int_{\omega=-\infty}^{\infty} e^{j \omega t} e^{-\frac{(\omega-\mu)^{2}}{2 \sigma^{2}}} \cdot d \omega
$$

the second integral is simply the same with $t$ replaced by $-t$. Consider the integrand

$$
e^{\frac{-(\omega-\mu)^{2}+j 2 \sigma^{2} \omega t}{2 \sigma^{2}}}
$$

This can be configured into the form of eqn. (9).

$$
e^{j \mu t} \cdot e^{-\frac{\sigma^{2} t^{2}}{2}} \cdot e^{\frac{-\left(\omega-\mu-j \sigma^{2} t\right)^{2}}{2 \sigma^{2}}}
$$




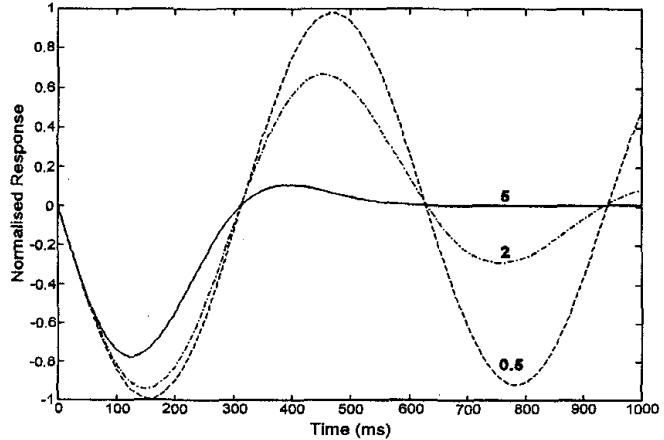

Fig. 3 Graph of $\mathrm{y}(\mathrm{t})$ for $\mathrm{n}=1, \mu=10$ and $\sigma=0.5,2 \& 5$, as shown.

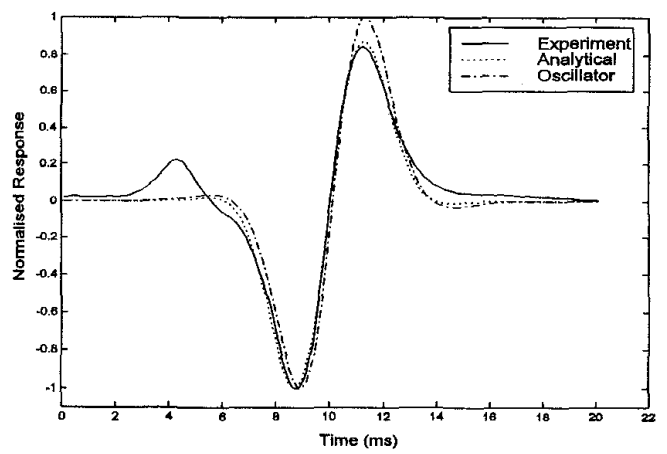

Fig. 4 Experimental (solid) and simulated oscillator assembly (dashed) and analytical (dotted) $\mathrm{H}$-wave responses.

$$
\therefore I_{1}=e^{j \mu t} \cdot e^{-\frac{\sigma^{2} t^{2}}{2}} \frac{1}{\sigma \sqrt{2 \pi}} \int_{-\infty}^{\infty} e^{\frac{-\left(\omega-\mu-j \sigma^{2} t\right)^{2}}{2 \sigma^{2}}} \cdot d \omega
$$

which from eqn. (9) reduces to

$$
\begin{aligned}
I_{1} & =e^{j \mu t} \cdot e^{-\frac{\sigma^{2} t^{2}}{2}} \\
\therefore \quad y(t) & =-e^{\frac{-\sigma^{2} t^{2}}{2}} \cdot \sin \mu t
\end{aligned}
$$

This is the result for resetting all the phases of the oscillators to zero. For $n$ oscillators the expected sum will be

$$
y(t)=-n \cdot e^{\frac{-\sigma^{2} t^{2}}{2}} \cdot \sin \mu t
$$

This function is graphed in Fig. 3 for $\mu=10$ and a range of $\sigma$ values. It is this oscillator network $y(t)$ that models the EMG signal observed during the $\mathrm{M}$ and $\mathrm{H}$-wave responses of the muscle. An interesting feature of the expression for $y(t)$ in eqn. (13) is that is solves the following equation describing a time-variant system [8].

$$
\frac{d^{2} y}{d t^{2}}+2 \sigma^{2} t \frac{d y}{d t}+\left[\sigma^{2}+\mu^{2}+\sigma^{4} t^{2}\right] y=n \mu \partial(t)
$$

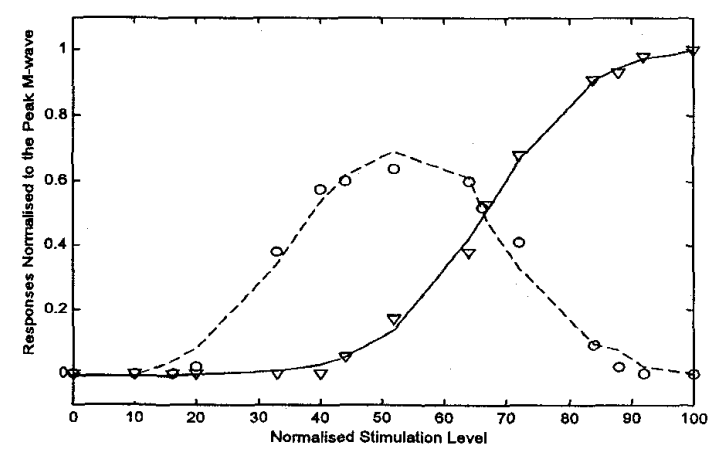

Fig. 5 Simulated M-wave (solid) and H-wave (dashed) recruitment curves. Data obtained in experiment for the $\mathrm{M}$-wave (open triangles) and the H-wave (open circles) are also shown.

where $\partial(t)$ is the unit impulse. The $\mathrm{M}$ - and $\mathrm{H}$-waves observed during a $\mathrm{H}$-reflex study has been modelled by assuming that our measurement system observes the outputs this community of oscillators reset in response to the electrical stimulus. Thus we have a model for EMG, where

$$
E M G=y(t)
$$

\section{RESULTS}

\section{Oscillator and Analytical Models}

Using a 2-D assembly of $20 \times 20$ oscillators a simulated response similar to the experimentally measured Hoffmann reflex is observed in the oscillator assembly upon resetting the oscillator phases in response to an electrical stimulation pulse. This response is shown in Fig. 4 where it is compared with a typical experimentally measured M-wave. The response obtained from the analytical representation of equation (13) is also shown. From the results in Fig. 4 it may be seen that both the oscillator assembly and analytical models presented here capture well the dynamics of the EMG measured from the muscle during an $\mathrm{M}$-wave.

\section{$H$ - and M-wave Recruitment Curves}

It is possible to construct a plot of the amplitude of the Mand $\mathrm{H}$-wave responses as a function of increasing stimulus intensity. These curves for the model presented here, as captured by the simple recruitment and spinal dynamics of equations (1) \& (2), are illustrated in Fig. 5, as are the values identified from experiment. The experimental results were obtained by performing the H-reflex test for different stimulus intensities, in accordance with Hugon's procedure [5]. From these data it may be seen that the model captures well the recruitment variations observed in experiment. 


\section{Total Model Behaviour}

Using the complete simulation model outlined in Fig. 2 results were obtained for the model's response to the stimulation pulses. These results are shown in Fig. 6 for stimulus amplitudes ranging from $0-100 \%$, in $10 \%$ steps, of the intensity required for the maximal $\mathrm{m}$-wave response.

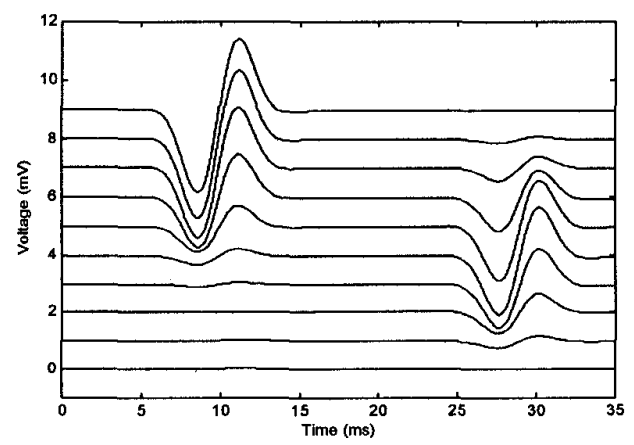

Fig. 6 Simulated control Hoffmann responses evoked at $10 \%$ intervals of max. stimulation intensity (standing upright). For clarity each test is shifted $3 \mathrm{mV}$ more along the Voltage axis.

A comparison of the simulation results presented in Fig. 6 with the experimentally determined data in Fig. 1 shows that that model presented here captures the Hoffmann reflex response dynamics observed in experiment.

\section{DISCUSSION}

The Hoffmann reflex is an important clinical tool for the investigation of neurological damage. In this paper a simple model of the Hoffmann reflex has been presented that may allow the quantification of changes observed in the reflex and their indication of the severity of spinal cord injury.

In Fig. 4 and 5 it was shown that this model, while simple, does capture the $\mathrm{M}$-wave EMG response and the M-wave and $\mathrm{H}$-wave recruitment curves observed in experiment. The shape of these $\mathrm{H}$ - and $\mathrm{M}$-wave recruitment curves may be used in the diagnosis of the inhibitory and facilitory nature of supraspinal control. Similarly, in Fig. 6 it was seen that this model could capture the Hoffmann response dynamics observed in experiment for varying levels of stimulation intensity.

The results presented in Fig. 4 showed a difference between the abilities of a $20 \times 20$ group of oscillators and the analytical model in capturing the experimentally determined M-wave response. This difference can be ascribed to the limited number of oscillators used and their limited resolution of the random variable $\omega$, whereas the analytical response is theoretically of infinite resolution.

Besides the simplicity of the analytical model over the oscillator network, identifying the parameters $\sigma$ and $\mu$ in equation (13) this model also provides the possibility of measuring the variance of the oscillator frequencies. This may provide a simple methodology for identifying gross changes in muscle fibre type and dynamics in muscle following spinal cord injury.

It has been shown that the ratios of the maximum Hoffmann Reflex of the control to swing and control to stance may be indicative of the severity of spinal injury, offering a measure of the integrity of the neurological control system [6]. This model affords a simple and swift way of analysing these levels of control.

\section{CONCLUSION}

A simple model for the Hoffmann reflex has been presented. A novel EMG model based on the synchronisation of a group of damped oscillators has been shown to be capable of producing an EMG response similar to that evoked during the Hoffmann Reflex by electrical stimulation of the posterior tibial nerve. A simpler analytical representation of this oscillator configuration has also been presented. This analytical model provides a means to calculate the variance of the underlying neurological oscillator model, which may be used as a benchmark for neurological studies.

\section{REFERENCES}

[1] J. Kimura, Electrodiagnosis in Diseases of Nerve and Muscle: Princples and Practice, F.A.Davis, Philadelphia, 1983.

[2] M. Schieppati, "The Hoffmann Reflex: A means of assessing spinal reflex excitability \& its descending control in man", Prog. Neurobiol., vol. 28, pp345-376, 1987.

[3] N. Bathien, A. Hugelin, "Reflexes Monosynaptique et Polysynaptiques chez l'homme au cours de l'attention". Electroenceph. Clin. Neurophysiol., vol. 26, pp. 604-612, 1969.

[4] C. J. Goodwill, "Locomotion: Analysis of gait, normal and abnormal", in Rehabilitation of the Physically Disabled Adult, Ed.: C. J. Goodwill and M.A. Chamberlain, Croom Held Ltd., London.

[5] M. Hugon, "Methodology of the Hoffmann Reflex in Man", New Developments in Electromyography and Clinical Neurophysiology, vol. 3, pp 277 - 293, 1973.

[6] C.D. Murray, J.D. Delaney, A.M. de Paor and R.B Reilly "Stimulation, capture and display device for the hoffmann reflex", Innovation et Technologie en Biologie et Medicine, vol. 13, pp. 642-652, 1992.

[7] M. Garrett, A. Ireland, R. Luckwill, "Changes in excitability of the hoffmann reflex during walking in man", Physiological Society, 23P, 1984.

[8] R.B.Reilly, T.E.Ward \& A.M.dePaor, "An Uncoupled Oscillator Model for Evoked Potential Dynamical Modelling", Proc. 18th Int. IEEE EMBS Conf, 1996. 